rumson

glyndwhr

Glyndŵr University

Glyndŵr University Research Online

Advanced Materials Research Laboratory

Materials Science

$3-1-2012$

\title{
Magnetophoretic assembly and printing of nanowires
}

\author{
Andrew C. Wright \\ Glyndwr University, a.wright@glyndwr.ac.uk \\ Michael Faulkner \\ University of Manchester
}

Follow this and additional works at: http://epubs.glyndwr.ac.uk/amrl

Part of the Bioelectrical and neuroengineering Commons, Engineering Science and Materials Commons, Nanoscience and Nanotechnology Commons, and the Other Materials Science and Engineering Commons

\section{Recommended Citation}

Wright, A \& Faulkner, M. (2012) “Magnetophoretic assembly and printing of nanowires."Journal of Vacuum Science \& Technology B, Vol. $30(2)$

This Article is brought to you for free and open access by the Materials Science at Glyndwir University Research Online. It has been accepted for inclusion in Advanced Materials Research Laboratory by an authorized administrator of Glyndŵr University Research Online. For more information, please contact d.jepson@glyndwr.ac.uk. 


\title{
Magnetophoretic assembly and printing of nanowires
}

\begin{abstract}
Nanowires are a well-established class of materials covering both metals and semiconductors. Although the synthesis of nanowires has been highly developed, their manipulation into specific device structures has lagged behind. Here we report a simple room temperature method for creating directly patterned structures out of nickel nanowires of submicron diameters as previously formed by electrotemplating. Specially shaped magnetic pole-pieces are used to form patterns of these ferromagnetic rods on thin flexible plastic foils, which were then fixed permanently into place either by electrodepositing an additional thin nickel coating or by a UV-curable polymer solution. It is shown that it is possible with oriented and patterned magnetic fields to create both vertically and, by using additional surface tension forces upon drying, horizontally aligned arrays of nickel rods. The authors show that linewidths down to $50 \mu \mathrm{m}$ can easily be realized with this technique and also show that direct printing of these magnetophoretically assembled structures onto adhesive or rubber substrates is also possible. The simplicity and low-cost inherent in this lithography-free method suggests that it is suitable as a general manufacturing method for nanowire assembly. As an example, a simple field emission display device is demonstrated.
\end{abstract}

\section{Keywords}

coatings, electrodeposition, ferromagnetic materials, magnetic thin films, metallic thin films, nanofabrication, nanomagnetics, nanopatterning, nanowires, nickel, polymer solutions, surface tension

\section{Disciplines}

Bioelectrical and neuroengineering | Biomedical Engineering and Bioengineering | Engineering | Engineering Science and Materials | Materials Science and Engineering | Nanoscience and Nanotechnology | Other Materials Science and Engineering

\section{Comments}

Copyright (C) 2012 American Vacuum Society, American Institute of Physics.

This article may be downloaded for personal use only. Any other use requires prior permission of the author and the American Institute of Physics.

This is the authors final version of the article. The following article appeared in Wright, A \& Faulkner, M. (2012) "Magnetophoretic assembly and printing of nanowires." Journal of Vacuum Science \& Technology B, Vol.30(2) and may be found at http://dx.doi.org/10.1116/1.3683152 


\title{
Magnetophoretic assembly and printing of
}

\section{nanowires}

Running title: Magnetophoretic assembly

\begin{abstract}
Andrew C Wright ${ }^{\text {a) }}$
Advanced Materials Research Laboratory, Materials Science Department, Glyndwr University, Wrexham, LL11 2AW, U.K.
\end{abstract}

Michael Faulkner ${ }^{\text {b) }}$

Department of Materials Science, University of Manchester, Grosvenor Street, Manchester, M13 9PL, U.K.

a) American Vacuum Society member.

a) Electronic mail: a.wright@glyndwr.ac.uk

b) Electronic mail: m.faulkner@manchester.ac.uk

a) Work tel. +1978293369

b) Work tel. +161 3065988 


\section{ASTRACT}

Nanowires are a well-established class of materials covering both metals and semiconductors. While the synthesis of nanowires has been highly developed, their manipulation into specific device structures has lagged behind. Here we report a simple room temperature method for creating directly patterned structures out of nickel nanowires of sub-micron diameters as previously formed by electrotemplating. Specially shaped magnetic pole-pieces are used to form patterns of these ferromagnetic rods on thin flexible plastic foils and which were then fixed permanently into place either by electrodepositing an additional thin nickel coating or by a UV-curable polymer solution. It is shown that it is possible with oriented and patterned magnetic fields to create both vertically and, by using additional surface tension forces upon drying, horizontally aligned arrays of nickel rods. We show that line widths down to 50 microns can easily be realized with this technique and also show that direct printing of these magnetophoretically assembled structures onto adhesive or rubber substrates is also possible. The simplicity and low-cost inherent in this lithography-free method suggests that it is suitable as a general manufacturing method for nanowire assembly. As an example, a simple field emission display device is demonstrated. 


\section{INTRODUCTION}

Nanowires are a well-established class of materials covering both metallic and semiconductive forms. Their synthesis is now quite highly developed and several reviews of the various methods now in use have been published by Wang et al. ${ }^{1}$ and by He and $\mathrm{Tao}^{2}$. In addition, carbon nanotubes form another class of one-dimensional materials and a review of the various methods of their synthesis has been published by Teo et al. ${ }^{3}$. Because of the unique properties often associated with 1D materials, it is desirable to fabricate nanowires into a variety of device structures and Li et al. ${ }^{4}$ have described the formation of both electronic and optoelectronic devices.

While some nanowires synthesis techniques form the material in-situ, it is by often using a method associated with considerably elevated temperatures such as Chemical Vapour Deposition (CVD) or similar. These techniques, apart from the energy costs involved, do not lend themselves to the creation of device structures on low-cost substrates such as plastics, elastomers or glass which have low thermal stability. Hence in this case it is advantageous to use some methods which can manipulate free-form nanowires into the desired pattern or form and then fix them in place. With this in mind, two methods have been described using either oriented electric or magnetic fields. For materials with a sufficiently strong dielectric response, the phenomena of dielectrophoresis has been used to create silicon interconnects as described by WissnerGross $^{5}$ and carbon nanotube structures by Bannerjee et al. ${ }^{6}$. Similarly, nanowires which are ferromagnetic in nature, or have ferromagnetic ends, can be attracted to and oriented 
by, magnetized tracks patterned onto a flat substrate as shown by Hangarter and Myung ${ }^{7}$ in the case of segmented nanowires viz. Ni-Au-Ni or in the case of $\mathrm{Ni}-\mathrm{ZnO}-\mathrm{Ni}$ as demonstrated by Lee et al. ${ }^{8}$ showing that semiconductor wires may be directly positioned in this way. However, both these methods require that pre-patterned electrodes be formed on the substrate in question using the usual lithographic techniques. The need for lithography naturally raises the cost of manufacture and thus we seek a cheaper means of patterning nanowires. Also, the use of patterned electrodes naturally means that the nanowires are to be formed only in-plane.

\section{A. Manufacturing vertical nanostructures}

However, the creation of vertically aligned nanowires (or microstructures) is desirable for many advanced applications such as field emission devices and displays [9], capacitor structures ${ }^{10}$, electrical vias in microcircuits ${ }^{11}$, micro-electro-mechanical structures (MEMS) ${ }^{12,13}$ and Lab-on-a-Chip ${ }^{14}$. Current methods for realising such vertical structures often center around either some type of etching process that favours the anisotropic removal of material from a flat substrate such as reactive ion etching ${ }^{15,16}$ or a process that directly builds up a vertical structure by selective deposition such as that used for carbon nanotube arrays ${ }^{17}$. In the case of carbon nanotubes, a catalyst is used in combination with CVD to create vertical fibers either by steric crowding as when singlewalled nanotubes are produced ${ }^{18}$ by thermal CVD or by the electric field induced alignment of multi-walled nanotubes obtained by plasma-assisted CVD process, PA$\mathrm{CVD}^{3}$. With PA-CVD, the potential drop across the plasma sheath directs the incoming carbon bearing species which has the effect of producing aligned fibers where small catalyst islands are present. Both CVD methods have an obvious drawback of requiring 
high temperatures for deposition of high quality material, typically at least several hundred degrees Celsius, making it incompatible with many other materials such as most plastics.

Deep (several mm), high aspect ratio (1000:1) vertical structures can be created by a combination of $\mathrm{x}$-ray lithography and electrodeposition, the so-called LIGA process $^{19}$. A similar approach can be performed with exposure to UV light instead of Xrays if lower aspect ratio, less precise, structures can be accepted. A disadvantage of using X-ray's is the long (several hours) exposure time required with PMMA resists and the need for access to synchrotron radiation.

Electrodeposition has been successfully used to synthesize linear microstructures by using a template or mould previously created by anodisation of aluminium films followed by in-filling with metal or metal oxide (electroforming) and subsequent removal of the template by chemical etching ${ }^{20}$. The quality of the wires formed in this way will depend on the exact process used but optoelectronic-quality $\mathrm{ZnO}$ has been realized by this technique ${ }^{8}$. Here we show an extension of this electroforming method to realize vertically aligned microstructures by the use of patterned magnetic fields to marshal and align nickel nanowires, previously made by electroforming and fixing them in place by electrodeposition (electrobonding). 


\section{B. Lithography-free magnetophoretic assembly of vertical and horizontal structures}

It has been known for a long time that magnetic field patterns can be visualized with iron filings distributed onto a flat surface. The iron filings are attracted towards regions of strong flux. Thus by creating a magnetic field which is concentrated by suitable patterning of planar magnetic structures we can attract sub-micron sized magnetic particles or fibres to those regions. By interposing a thin foil, coated with a nonmagnetic conductive layer, between the magnetic structure and a dispersion of the particles in a liquid carrier, we can then fix these particles in place by over-plating them with a metallic electrodeposit. Alternatively, we can fix the nanowires in place using a UV-cured polymer/acetone mixture. With magnetic nano-rods such as those created by templating of nickel in porous alumina films ${ }^{21}$, the rods will aligned along the lines of magnetic flux, which with a suitably designed magnetic structure, will be normal to the plane of the substrate.

As described above, the method has already been used to create horizontal arrays of aligned magnetic nanowires using pairs of ferromagnetic electrodes pre-patterned onto the substrate ${ }^{7,8}$. To create vertically aligned nanowires the magnetic flux must emerge from the plane of the substrate and for patterning we must shape the magnetic field by the creation of a specially shaped magnetic pole-piece. 
To demonstrate this, the procedure used in our work uses a thin foil of ferromagnetic nickel formed into a simple ' $\mathrm{V}$ 'shape embedded in non-magnetic resin and is magnetized from the rear using a small permanent magnet. A thin metallized plastic foil is closely contacted to the front face of the magnetic head and immersed briefly in a dispersion of nickel nanowires in a slow drying solvent such as iso-propyl alchohol. The magnetic flux lines are normal to the plane of the substrate rather than in-plane as used previously and gives a surface coated with nickel nanowires highly concentrated in regions of enhanced magnetic flux i.e. above the now magnetized nickel ' $V$ '. The nanowires are expected to remain with the same alignment once fixed in place by either a thin nickel or a UV-cured polymeric overcoat.

Direct print transfer of nanowires from the bare magnetic head to an adhesive substrate also offers an appealing way of creating nanowires structures in a simple low cost way. Finally, by using a closed magnetic loop involving two closely spaced embedded magnetized foils (or wires), it may be possible to realize horizontal alignment of nanowires on a substrate.

\section{EXPERIMENTAL}

\section{A. Fabrication of nickel nanowires}

Nickel nanowires were made using the well-known template method ${ }^{22}$. The method appears to produce polycrystalline material rather than single crystals but this does not detract from its basic magnetic properties. Alumina membranes (Whatman 
Anopore), $47 \mathrm{~mm}$ diameter, with pores of 0.2 microns were used to create nanowires some 17-18 microns long and, after washing, ultrasonically dispersed in iso-propyl alchohol (IPA). The dispersion is stable for several minutes before the nanowires finally settle out.

\section{B. Fabrication of patterned magnetic head}

An initial trial of using laser ablation to create a patterned pole-piece or 'head' out of soft magnetic iron to concentrate the magnetic field from a permanent magnet into an ' $\mathrm{X}$ '-shape was unsuccessful as it was not possible to cut a sufficiently high aspect ratio structure into the iron using this method; machined depths of only $0.5 \mathrm{~mm}$ were possible with this method. We therefore used a thin nickel foil, 50 microns thick, formed into a ' $\mathrm{V}$ ' shape and potted with epoxy resin in a $12 \mathrm{~mm}$ diameter glass tube and then ground flat on both sides to a final thickness of between 4 to $10 \mathrm{~mm}$ (Supplementary Fig. S7a). This was bonded with wax (Aremco Crystalbond 509) to a $10 \mathrm{~mm}$ diameter, $5 \mathrm{~mm}$ thick 430 grade magnetic stainless steel disc which was magnetized with a single $25 \mathrm{~mm}$ long, 6 mm diameter Alnico V magnet, (Supplementary Fig. S7b) or, for higher power, a stack of $25 \mathrm{NdFeB}$ discs (Type EP643, diameter 6mm, thickness 4mm) both from E-Magnets UK Ltd. Thinner nickel foils were created from polyimide sheet (80 microns thick) first sputter coated with chromium (100nm) followed by electrodeposition to desired thickness of 5 microns and 16 microns. These were bent into simple ' $\mathrm{V}$ ' shapes and mounted in resin as above. 


\section{Formation of patterned nanowire structures}

A $15 \mathrm{~mm}$ by $15 \mathrm{~mm}$ square thin ( 80 micron) foil of Kapton polyimide, sputter coated with 100nm of chromium, was bonded using rubber adhesive or Crystalbond wax to the front face of the ' $\mathrm{V}$ ' and a $4 \mathrm{~mm}$ wide thin strip of the foil was brought around the side of the assembly for electrical contacting (Supplementary Fig. S7c). To improve electrical conductivity for electroplating, the strip and edges of the Kapton were coated with colloidal silver paint.

Two magnetic structures were explored. The first, 'single magnet', used a single magnet assembly as described above and the second 'double magnet' using a second identical Alnico magnet (not fitted with a magnetic steel disc) held in a separate clamp some $5 \mathrm{~mm}$ away from and above the foil face with 'North' facing 'South' to provide flux lines more close to the foil normal.

The foil face of the combined single magnet structure was brought into contact with the IPA dispersed nanowires for 10 seconds. To ensure a level of consistency in the density of nanowires collected, a delay of 20 seconds was given after the ultrasonic dispersion was stopped. Initially, the foil was allowed to dry before immediate immersion of the foil face in a standard composition ${ }^{23}$ room-temperature sulfamate-based nickel electroplating bath run at $10 \mathrm{~mA}$ for 20 minutes. The thin chromium coating does not always provide sufficient conductivity for rapid plating and could be enhanced with a copper or preferably a gold overcoat. However, chromium does adhere quite well to the 
Kapton strip when bent backwards to make the electrical contact. As an interim, we used colloidal silver paint as described above to reduce the circuit resistance.

The above was repeated using a double magnet assembly by bringing the magnet and disc assembly under the second magnet before the dispersion has time to dry.

As an alternative to fixing in place by over plating, we used a UV-curable optical adhesive (Norland 60, Edmund Optics Ltd.) which had been diluted with acetone (1-3 drops of adhesive to $4 \mathrm{ml}$ of acetone). The patterned foil was immersed in the solution and allowed to dry. Curing was done with a 200W Hg lamp fitted with flexible liquid light guides. It was found that monochromated light at $365 \mathrm{~nm}$ was insufficient to achieve full cure and so the full broadband output of the lamp was used.

\section{Use of adhesive substrates}

We used double sided electrically conductive pads as used for mounting SEM specimens on stubs (Agar Scientific). The magnetic heads were given a thin coating of light oil as a release agent prior to decoration with nanowires. The head with attached nickel nanowires was simply pressed onto the pad and pulled away leaving the nanowires adhered. Silicone rubber (Aremco Ezi-Cast 580) was used to fully embed nanowires before curing at ambient temperature. Partly cured rubber (five hours after mixing the two components) was similar to the adhesive pads except that no release agent was necessary to facilitate removal of the magnetic head. We used a strong NdFeB magnet 
(Type EP376, diameter $10 \mathrm{~mm}$, length $20 \mathrm{~mm}$ ) with partly cured rubber to realign the nanowires before full cure took place.

\section{E. Finite Element Modelling (FEA) of magnetic fields}

The freely available 2D simulation package $\mathrm{FEMM}^{24}$ was used to model magnetic flux distribution. Briefly, a circular field of diameter $200 \mathrm{~mm}$ was used to contain the model. Edge effects were taken into account using the Kelvin Transformation. While the Alnico magnet and steel disc were modeled with a mesh of $0.25 \mathrm{~mm}$, the very thin $(50$ microns) nature of the nickel foil made it necessary to employ a much finer mesh of $0.003 \mathrm{~mm}$. In addition, a finer sampling box was created around the end of the $\mathrm{Ni}$ foil with a mesh size of $0.001 \mathrm{~mm}$. The main circular field used a mesh size of $2 \mathrm{~mm}$ and this was reduced in stages with successive boxes of $0.1 \mathrm{~mm}$ and $0.01 \mathrm{~mm}$ mesh spacing towards the edge of the nickel foil. Typically, a total of 155744 nodes and 310340 elements were used in the finite element calculation. The Alnico 5 magnet (Anisotropic Cast type, part \# EP276 from E-Magnets UK ltd., grade ACA40) had a listed strength of 1.20 Tesla and a coercivity of $50 \mathrm{kA} / \mathrm{m}$ while the NdFeB magnets (E-Magnets UK Ltd. part \# EP643 and EP376, grade N42) had listed strengths of 1.28T and a coercivity of 923 $\mathrm{kA} / \mathrm{m}$. 


\section{RESULTS AND DISCUSSION}

\section{A. Results}

Interestingly, we found that with a single Alnico V magnet assembly, the nanowires were usually oriented in the plane of the Kapton foil surface rather than with the vertical (Figure 1a,b and Supplementary Fig. S1). That the nickel nanowires could be seen optically to align vertically under microscopic examination before the IPA solvent dried strongly indicates that surface tension forces are able to overcome magnetic forces by pulling the fibers down onto the foil surface. We found that this was the case whether the solvent was water, IPA or acetone/monomer. The width of the nanowire array at 200 microns is typically four times that of the width of the nickel foil used for the magnetic head.

With the double Alnico V magnet assembly, the nickel nanowires remain vertical, following the flux lines, during the solvent evaporation stage, showing that the magnetic forces are now strong enough to resist surface tension forces and remain upright during the fixing stage, Figure 2a where a nickel overplate has been used to fix the nanowires in place (See also Supplementary Fig. S2 where UV-cured adhesive has been used to embed the nanowires in a vertical alignment). Again, the width of the nanowire array at 200 microns is some four times that of the underlying nickel foil head. Stray fields from the backing magnet cause nanowires to be attracted to areas outside the ' $\mathrm{V}$ ' area when shallow $(4 \mathrm{~mm})$ heads are used. This effect can be reduced by using deeper $(10 \mathrm{~mm})$ heads. 
Fig. $2 \mathrm{~b}$ shows a more detailed view of the metal plated nanowires where it can be seen that nanowires are clumped together in bundles. Figure $2 \mathrm{~b}$ also shows that bundles of nanowires often stack end to end making longer vertical structures (See also Supplementary S3). These figures shows that the individual nanowires are some 18 microns long and that the electrodeposited nickel overcoat has increased the nanowire diameter to about 2 microns, some ten times the original diameter. However, an examination of free nanowires after release from the alumina template shows that the bundling is a direct result of incomplete separation of individual nanowires, most probably caused by cracks in the template allowing thin bridges to form between nanowires.

To explain the observation of the nanowires lying across the plane of the nickel foil when created with the single magnet assembly, we modeled the magnetic field flux using a simple 2-D approach. With both the single and double magnet assemblies, we find that flux density maps show that magnetic flux is concentrated at the corner edges of the nickel foil (Supplementary Fig. S4). However, at the point corresponding to the substrate surface, this effect disappears with a gradual peak in the flux density in the centre (See Supplementary Fig. S5).

Therefore lateral alignment must arise from other effects and we subsequently performed an experiment to observe the nanowires under a stereo microscope during the evaporation of the solvent. The solvent first evaporates from the nanowire-free areas leaving a linear array of vertically aligned nanowires immersed in solvent. With a single 
magnet assembly, the magnetic field lines diverge away from the end of the $\mathrm{Ni}$ foil and this appears to bias the orientation of the nanowires across its width. With a stronger rareearth magnet which provides enough vertically aligning force to overcome surface tension on drying, SEM images show the diverging nature of the field (Supplementary Figure S6) as indicated by the changing orientation of vertically aligned nanowires from inside to outside of the' $V$ '. This was confirmed by observation of the drying process whereby nanowires could be seen to be well aligned vertically under the solvent but were gradually pulled over horizontally by the effect of surface tension as the solvent evaporated away. With heavier deposits of nickel nanowires, we also found that they would suddenly snap to vertical orientation after the solvent had evaporated indicating that dispersion forces holding fibers to lie in-plane can be overcome if sufficient magnetic material is present.

We found it best to avoid drying forces altogether by washing the formed nanowire array with water (IPA solvent causes the nickel sulfamate to crystallize out) and then immediately transferring the assembly to the plating bath while still wet. We found that vertically aligned nanowire arrays could then easily be formed.

However, with the double magnet assemblies, the magnetic flux strength is much greater giving vertical alignment by overcoming surface tension forces upon drying. The force of attraction however experienced by the nanowire towards the substrate arises from flux density gradients at that point via the following equation: 


$$
F=\frac{\partial B}{\partial x} \cdot \mu
$$

Where $\mu$ is the magnetic moment of the nanowire and B is the flux density.

By contrast, the torque force of alignment arises from the absolute value of flux density acting on the magnetic moment via the vector product:

$$
T=B \times \mu
$$

Or in terms of a misalignment angle from the lines of flux we have:

$$
T=B \cdot \mu \cdot \operatorname{Sin} \theta
$$

Any magnetic arrangement for vertical alignment of nanowires should therefore have a high enough flux gradient to achieve attraction to the surface and enough absolute flux density to maintain that alignment during drying. In practice this seems best achieved by using a double magnet structure. Alternatively, a single electromagnet working within a closed loop magnetic structure having an air gap could be employed. Using finite element modeling, we find that the flux density at the plane of the substrate 
for the single Alnico magnet structure is $0.068 \mathrm{~T}$ while that for the double Alnico magnet assembly is $0.103 \mathrm{~T}$. These figures rise to $0.158 \mathrm{~T}$ and $0.385 \mathrm{~T}$ when stronger $\mathrm{NdFeB}$ magnets are used with the same dimensions. The flux gradients are $-0.35 \mathrm{~T} / \mathrm{mm}$ for single Alnico magnets at the plane of the substrate and rises to $-0.46 \mathrm{~T} / \mathrm{mm}$ for the double Alnico magnet configuration. When stronger NdFeB magnets of similar size are used these values rise to $-0.56 \mathrm{~T} / \mathrm{mm}$ and $-0.62 \mathrm{~T} / \mathrm{mm}$ respectively. Stronger field gradients give rise to the formation of denser nanowire arrays for a given immersion time in the dispersion. We calculate that a flux gradient of at least $0.0048 \mathrm{~T} / \mathrm{mm}$ is required to attract a single nickel nanowire immersed in iso-propyl alcohol (see Supplementary) and our flux gradients are clearly much greater than this.

We have greater confidence in the predicted values for the $\mathrm{NdFeB}$ magnet as Alnico materials are notoriously difficult to model accurately $y^{25}$ having inhomogeneous demagnetization characteristics. Therefore the predicted figures for the Alnico V configurations are likely to be too high. A stock magnet was measured using a Gaussmeter to have a strength of $14.8 \mathrm{mT}$ at a point $8 \mathrm{~mm}$ away from the end, about half that predicted by simulation.

We explored the potential for this method to address narrower line widths using thinner nickel foils. Correspondingly, to avoid field divergence reducing resolution, we reduced the thickness of the substrate to 14 microns by using polyester film (Mylar) sputter coated with Cr. The results are shown in Fig. 3a-d for 16 microns and 5 microns thick nickel foil heads where the width of the nanowire arrays are some 100 and 50 
microns respectively. It is possible even narrower line widths could be achieved with further optimization.

We then explored the potential for direct printing from the pole-piece to a substrate. Nanowires were then directly printed onto double sided adhesive discs, Fig. 4a, or onto partly cured silicone rubber, Fig. 4b,c.

The results of using a UV-curable optical adhesive (1 drop adhesive in $4 \mathrm{ml}$ acetone) are shown in Fig. 5a,b which show strong clumping into triangular shapes as the acetone evaporates rapidly. This is in contrast to figure 1 where a higher concentration of adhesive was used (4 drops in $1 \mathrm{ml}$ acetone), effectively embedding the nanowires.

Finally, as an initial example of an application of this technology, we constructed a simple device to demonstrate the potential for field emission from the vertically aligned nickel nanorods. A simple test jig was constructed (Supplementary S8(a)) using a 'V' structure made with a 50 micron nickel foil (cf. Fig. 2). The vacuum gap varies considerably because of large variations in the total stacked nanorod height and this is reflected in the observation of only discrete points of light (Supplementary S8(b)). Nickel has a high work function $(\Phi)$ of between 5.01 to $5.35 \mathrm{eV}$ and this result in a high extraction voltage $(\sim 2 \mathrm{kV})$. Vacuum overcoating of the nickel nanorods with a lower workfunction metal such as $\mathrm{Al}(\Phi \approx 4 \mathrm{eV})$ would reduce the operating voltage as would reducing the nanorod diameter by enhancing the field concentrating effect of a sharper tip. However, the most important issue to address for this application is to gain better control of the total nanorods height i.e. more uniform across the emitting area. 


\section{B. Discussion}

The method of using magnetic forces for creating vertical structures out of solvent-dispersed nanoparticulate material subsequently fixed in place with metal overplating or polymer adhesives has some advantages over standard CVD, RIE or lithographic methods in that it is done at room temperature using a very simple and cost effective method and is directly compatible with polymer substrates. It does require a prepatterned magnetic 'master' to be made first but like a CD stamp, can be re-used many times. The resolution of the technique depends in part on the feature size of the master but also on the distance between it and the surface to be coated as the flux lines will tend to diverge unless a closed magnetic circuit is used. This is less of an issue if transfer printing directly from the magnetic head onto a substrate is to be used. With the use of a closed magnetic circuit, potentially high resolution could be achieved as it is possible to synthesize much smaller diameter nickel nanowires either by using templates with smaller pore sizes or by chemical synthesis ${ }^{26}$.

While the vertically oriented metal nanowires can be disturbed or bent by direct contact, the structures created using this technique are quite robust to handle in terms of repeated flexing of the plastic substrate foils, being only limited by the adhesion of the initial metallization layer. The application of a strong and ductile overplated metallization is clearly very helpful for ease of handling and this is generally facilitated by many plating processes. 
The method has obvious potential for creating horizontal vias between conductive pads by exploiting the way surface tension forces combined with a slightly angled magnetic field can orient the nanowires directly across the gap. When combined with nanowires made as multilayers with magnetic ends ${ }^{7,8}$, sensor elements of semiconductive or gas-sensitive metals may readily be constructed. It may even be possible to create strain gauge devices from suitable materials in this way if the ends can be fixed in place. The technique may also be usefully combined with the recent development of semiconductor nanowire based photovoltaic cells ${ }^{27}$.

While expensive, LIGA has the capability to make very deep high aspect ratio nickel structures for the magnetic master. Alternatively, the use of micro-extrusion or rapid prototyping (3D printing) of plastic formers with vacuum coating and electrodeposition can in principle create arbitrarily shaped magnetic heads. With optimization and further development, magnetic data storage structures may be realized by this means. As a low cost means of creating field emitting structures, it does have some considerable merit as these structures can be formed entirely at room temperature on any material given a conductive coating. It remains to be seen whether individual nanowires can be accurately placed by this method if a sufficiently very small magnetized region for the head could be created via the LIGA technique. 
The observed end stacking of nanowires is clearly undesirable where constant height structures are required viz. Field Emission. Lower field gradients may be needed to ensure that vertical arrays only one nanowire length deep are formed and further optimization is required to attain this.

\section{IV.SUMMARY AND CONCLUSIONS}

By using specially shaped magnetic pole-pieces we have shown that it is possible to directly pattern magnetic nickel nanowires onto thin plastic substrates with a line resolution down to at least 50 microns which can then be fixed in place using either a thin electrodeposited metal or a UV-cured polymer. We have also showed that direct transfer of the nanowires from the magnetic patterned head to adhesive substrates can also be achieved. Both vertical and, when combined with surface tension effects, horizontal alignment of the nanowires is possible with this method. We have shown that a simple field emission device can be constructed using this methodology. The technique has promise for lithography-free printing of nanowires-based structures for devices based on them and deserves further development. 


\section{ACKNOWLEDGMENTS}

The authors would like to thank Dr. Euan Goodier of E-Magnets UK Ltd. for provision of data on the various magnets used in this research. ACW invented all the fabrication techniques used in this research and did the experiments. MF did all of the SEM imaging. The Authors declare no competing financial interests 


\section{References}

${ }^{1}$ N.Wang, Y. Cai and R.Q. Zhang

Mater. Sci. and Eng. R60, 1 (2008)

${ }^{2}$ H. He and N.J. Tao

Encyclopedia of Nanoscience and Nanotechnology, 2 edited by H. S. Nalwa,

(American Scientific Publishers 2011) Vol. 2, pp. 755-772

${ }^{3}$ K.B.K. Teo, C. Singh, M. Chhowalla and W.I. Milne

Encyclopedia of Nanocience and Nanotechnology, edited by H. S. Nalwa

(American Scientific Publishers 2004) Vol. 1, pp. 665-686

${ }^{4}$ Y. Li, F. Qian, J. Xiang, and C.M. Lieber Nanowire electronic and optoelectronic devices

Mater. Today 9(10), 18 (2006)

${ }^{5}$ A.D. Wissner-Gross

Nanotechnology 17, 4986 (2006) 
${ }^{6}$ S. Banerjee, B.E. White, L. Huang, B.J. Rego, S. O’Brien and I.P. Herman

J. Vac. Sci. Technol. B24 3173 (2006)

${ }^{7}$ C.M. Hangarter and N. Myung 2005

Chem. Mater. 17, 1320 (2005)

${ }^{8}$ S.W. Lee, M.C. Jeong, J.M. Myoung, G.S. Chae and I. J. Chung

Appl. Phys. Lett. 90, 133115 (2007)

${ }^{9}$ W.I. Milne, K.B.K. Teo, M. Chhowalla, G.A.J. Amaratunga, S.B. Lee, D.G. Hasko,

H. Ahmed, O. Groening, P. Legagneux, L. Gangloff, J.P. Schnell, G. Pirio, D. Pribat,

M. Castignolles, A. Loiseau, V. Semet and Vu Thien Binh

Diam. and Rel. Mater. 12, 422 (2003)

${ }^{10}$ J.E. Jang, S.N. Cha, Y. Choi, G.A.J. Amaratunga, D.J. Kang, D.G. Hasko, J.E. Jung and J.M. Kim

Appl. Phys. Lett. 87, 263103 (2005) 
${ }^{11}$ F. Kreupl, A.P. Graham, M. Liebau, G.S. Duesberg, R. Seidel and E. Unger

IEEE International Electron Devices Meeting, Technical Digest 683 (2004)

${ }^{12}$ C. Hierold, A. Jungen, C. Stampfer and T. Helbling

Sens. and Actuators A 136, 51 (2007)

${ }^{13}$ J.E. Jang, S.N. Cha, Y.J. Choi, D.J. Kang, T.P. Butler, D.G. Hasko, J.E. Jung, J. M.

Kim and G.A.J. Amaratunga

Nat. Nanotechnol. 3, 26 (2008)

${ }^{14}$ C.L. Choong, W.I. Milne and K.B.K. Teo

Int. J. Mater. Forum 1, 117 (2008)

${ }^{15}$ H. Janseny, H. Gardeniers, M. de Boer, M. Elwenspoek and J. Fluitman

J. Micromech. Microeng. 6, 14 (1996)

${ }^{16}$ Y.Q. Fu, A. Colli, A. Fasoli, J.K. Luo, A.J. Flewitt, A.C. Ferrari and W.I. Milne

J. Vac. Sci. Technol. B 27, 1520 (2009) 
${ }^{17}$ Z.F. Ren, Z.P. Huang, J.W. Xu, J.H. Wang, P. Bush, M.P. Siegal and P.N. Provencio Science. 282, 1105 (1998)

${ }^{18}$ K. Hata, D.N. Futaba, K. Mizuno, T. Namai, M. Yumura and S. Iijima

Science. 306, 1362 (2004)

${ }^{19}$ LIGA and its applications, ed. V. Saile, U. Wallrabe, O. Tabata and J. G. Korvink (Wiley-VCH Wernheim 2008) Vol. 7 Advanced Micro and Nanosystems series

${ }^{20}$ D. Routkevitch, A.A. Tager, J. Haruyama, D. Almawlawi, M. Moskovits and J.M. Xu IEEE Trans. Elec. Dev. 43, 1646 (1996)

${ }^{21}$ R.M. Metzger, V.V. Konovalov, M. Sun, T. Xu, G. Zangari, B. Xu, M. Benakli and W.D. Doyle

IEEE Trans. Magnet. 36, 30 (2000)

${ }^{22}$ A.K. Bentley, M. Farhoud, A.B. Ellis, G.C. Lisensky, A.M.L. Nickel and W.C. Crone J. Chem. Educ. 82, 765 (2005) 
${ }^{23}$ G.A. Bari ASTM Handbook (American Society for Testing of Materials, Ohio, 8th Edition 1994) Vol. 5 Surface Engineering, pp. 201-212

${ }^{24}$ FEMM Magnetics Finite Element Analysis package www.femm.com

${ }^{25}$ K.B. Hughes and D.I. Gordon

Power Eng. Rev. 7, 28 (1987)

${ }^{26}$ Z.G. Qiua and H.M. Menga

Mater. Chem. and Phys. 113, 227 (2009)

${ }^{27}$ J. Tang, Z. Huo, S. Brittman, H. Gao and P. Yang

Nature Nanotech. 6, 568 (2011)

${ }^{28}$ Handbook of Chemistry and Physics 78th ed. (CRC Press 1997-1998) 


\section{Figure Captions}

Figure 1. Magnetically patterned horizontally aligned nickel nanowires on polyimide foil. (a) SEM image showing ' $\mathrm{V}$ ' pattern created with horizontal nanowire alignment formed from the magnetic field from a ' $\mathrm{V}$ ' shaped thin nickel foil backed with a single Alnico magnet and (b) SEM close up view of nanowires showing they are aligned across the width of the edge of the magnetised nickel foil. The nanowires have been fixed in place with a UV-cured adhesive. Scale bars are $1 \mathrm{~mm}$ (a) and 50 microns in (b).

Figure 2. Magnetically patterned vertically aligned nickel nanowires on polyimide foil. (a) SEM image of patterned nickel nanowires created using a double Alnico magnet assembly on metallised polyimide sheet. A light electroplated nickel overcoat has been used to fix the nanowires in place. Both bundling and end to end stacking of nanowires is clearly shown (b). Scale bars are 500 microns in (a) and 50 microns in (b).

Figure 3. Higher resolution magnetophoretic assembly of nickel nanowires. (a) Nickel nanowires on polyester film (14 microns) formed from magnetized ' $\mathrm{V}$ ' made from 16 micron thick nickel foil. Vertical view (b) shows projected width of 100 microns. V pattern obtained with a 5 micron thick nickel foil magnetic head (c) shows a projected track width of around 50 microns (d). Scales bars are: 500 microns (a), 200 microns (b), 500 microns (c) and 200 microns (d). 
Figure 4. Direct printing onto adhesive substrates. Nickel nanowires transferred directly from magnetic head to a double sided adhesive SEM mount pad by contact printing (a) show flattened fibers while printing onto partly cured silicone rubber followed by realignment with strong NdFeB magnet (b), (c). Scale bar on SEM image (a) is 500 microns.

Figure 5. Vertically aligned nickel nanowires fixed in place with thin coat of UV-cured adhesive. Nickel nanofibers patterned onto polyimide (80 micron) using a ' $\mathrm{V}$ ' shaped 50 micron thin magnetised nickel foil and fixed in place using UV-cured polymer diluted in acetone (a). Note triangular clumping caused by surface tension forces on drying (b). Scale bars are $1 \mathrm{~mm}$ in (a) and 200 microns in (b). 